\title{
Explaining the Magnetic Properties of Oxygen Deficient LSMO Thin Films by iDPC
}

Aubrey N. Penn ${ }^{1}$, Robbyn Trappen ${ }^{2}$, Navid Mottaghi ${ }^{2}$, Chih-Yeh Huang ${ }^{2}$, Abinash Kumar ${ }^{1}$, Mikel Holcomb $^{2}$, and James M. LeBeau ${ }^{1 *}$

1. Department of Materials Science \& Engineering, North Carolina State University, Raleigh, NC, USA.

2. Department of Physics \& Astronomy, West Virginia University, Morgantown, WV, USA.

* Corresponding author: jmlebeau@ncsu.edu

The manganite family of perovskites are among the most promising materials in magnetoelectronics research due to their colossal magnetoresistance, room temperature ferromagnetism, and high curie temperature [1]. In thin films, however, a magnetic dead layer (MDL) arises, diminishing the magnetic properties in isolated regions [2]. The origin of the MDL has been widely studied in the context of oxygen vacancies or cation intermixing to accommodate for the interfacial polar discontinuity, strain due to lattice mismatch with the substrate, and electronic reconstruction by octahedral distortion or rotation. Currently no conclusion about the MDL origin is established, but there is evidence that the answer lies in the oxygen octahedral structure [3].

In this presentation, we quantify the local octahedral structure in PLD-grown $\mathrm{La}_{0.7} \mathrm{Sr}_{0.3} \mathrm{MnO}_{3}$ (LSMO) on STO under oxygen-stoichiometric and oxygen-deficient growth conditions using scanning transmission electron microscopy (STEM). The structure is correlated to magnetization measured with pulsed neutron reflectivity (PNR). Figure 1(a) shows the magnetization profile of the oxygen deficient film revealing an MDL 5 unit cells into the film. Previous studies suggest oxygen vacancies as the primary compensation mechanism for polar interfaces, however our chemical analysis performed via energy dispersive X-ray spectroscopy (EDS), Figure 1(b, c), shows approximately 2 unit cells of Ti intermixing into the film in both stoichiometric and oxygen deficient films. This is evident not only by the presence of Ti signal across the interface, but also by the loss of Mn signal in the same region. Results from EELS show that Ti remains $4+$ across the interface, and there is no evidence of a change in the Mn charge state.

To measure the oxygen atom column positions directly, we apply integrated differential phase contrast (iDPC) to observe the oxygen sub-lattice simultaneously with the heavier cations [4]. To remove drift and scan distortion, we apply the Revolving STEM (RevSTEM) method [5] to simultaneously acquire ADF and iDPC images. Figures 2(a-d) show resulting datasets for the stoichiometric and oxygen deficient LSMO thin films grown on STO. Based on analysis of the structure, we will demonstrate that the octahedral distortion depends strongly on the oxygen content during growth. Specifically, the iDPC images reveal that the oxygen columns are found to alternate in shape between round and elliptical for the oxygen deficient sample in contrast to the stoichiometric film. To further explore the distortion, we will show how out-of-plane octahedral distortion is drastically different for the two films, Figures 2(e, f). The stoichiometric film shows consistent, largely positive distortion that relaxes away from the interface, while the oxygen deficient LSMO film exhibits reduced distortion, primarily in the opposite direction. In both cases, the in-plane distortion, shown in Figures $2(\mathrm{~g}, \mathrm{~h})$ remains mostly flat, but the standard deviations of the distortions are 0.39 and $0.24 \mathrm{pm}$ for the oxygen deficient and stoichiometric films, respectively. This further confirms the higher degree of distortion in the oxygen sub-lattice for the oxygen deficient film.

Overall, we find the origin of the dead layer to not be easily explained by one phenomenon, but rather by a combination of intimately connected atomic and electronic structural characteristics that can be 
investigated by combining spectroscopy and light element imaging by iDPC.

References:

[1] S. Jin, T. H. Tiefel, M. McCormack, et al., Science 264 (1994) p. 413.

[2] M. Huijben, L. W. Martin, Y.-H. Chu, et al., Physical Review B 78 (2008) p. 094413.

[3] Z. Liao, M. Huijben, Z. Zhong, et al., Nature Materials 15 (2016) p. 425.

[4] I. Lazić, E. G. T. Bosch, S. Lazar, Ultramicroscopy 160 (2016) p. 265.

[5] X. Sang, J. M. LeBeau, Ultramicroscopy 138 (2014) p. 28.

[6] This work is supported by the National Science Foundation (DMR-1608656). The authors also acknowledge the Analytical Instrumentation Facility (AIF) at North Carolina State University, which is supported by the State of North Carolina and the National Science Foundation.
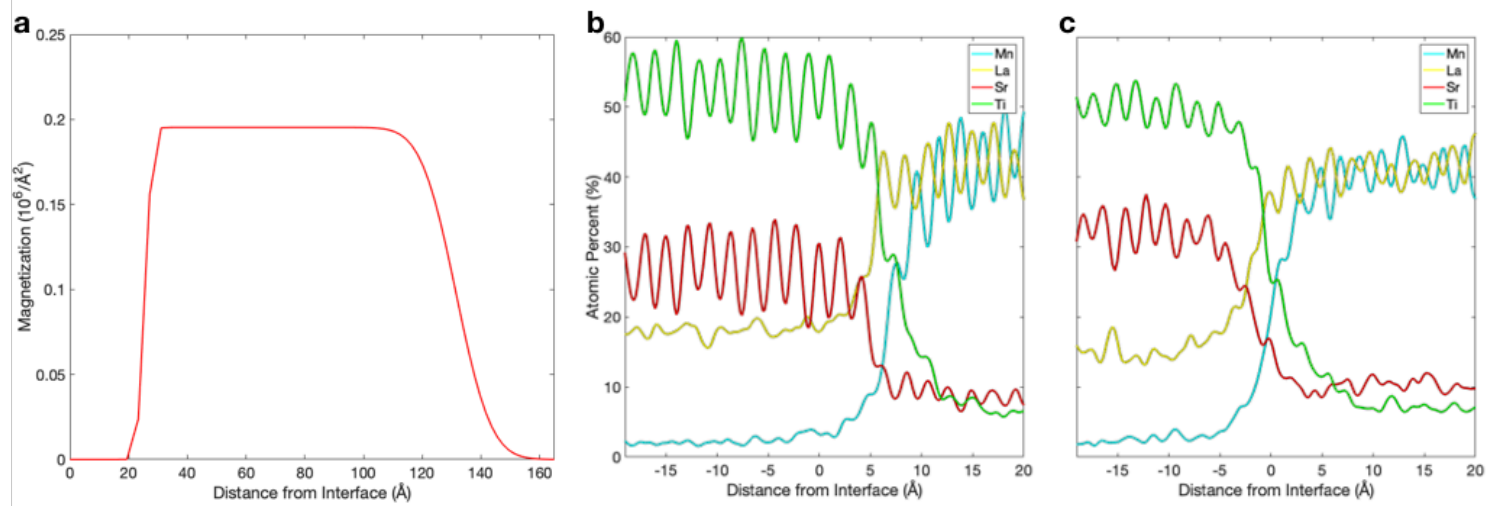

Figure 1. (a) Magnetization profile of the oxygen deficient film determined by pulsed neutron reflectivity. EDS line profiles of the oxygen deficient (b) and stoichiometric (c) LSMO films.

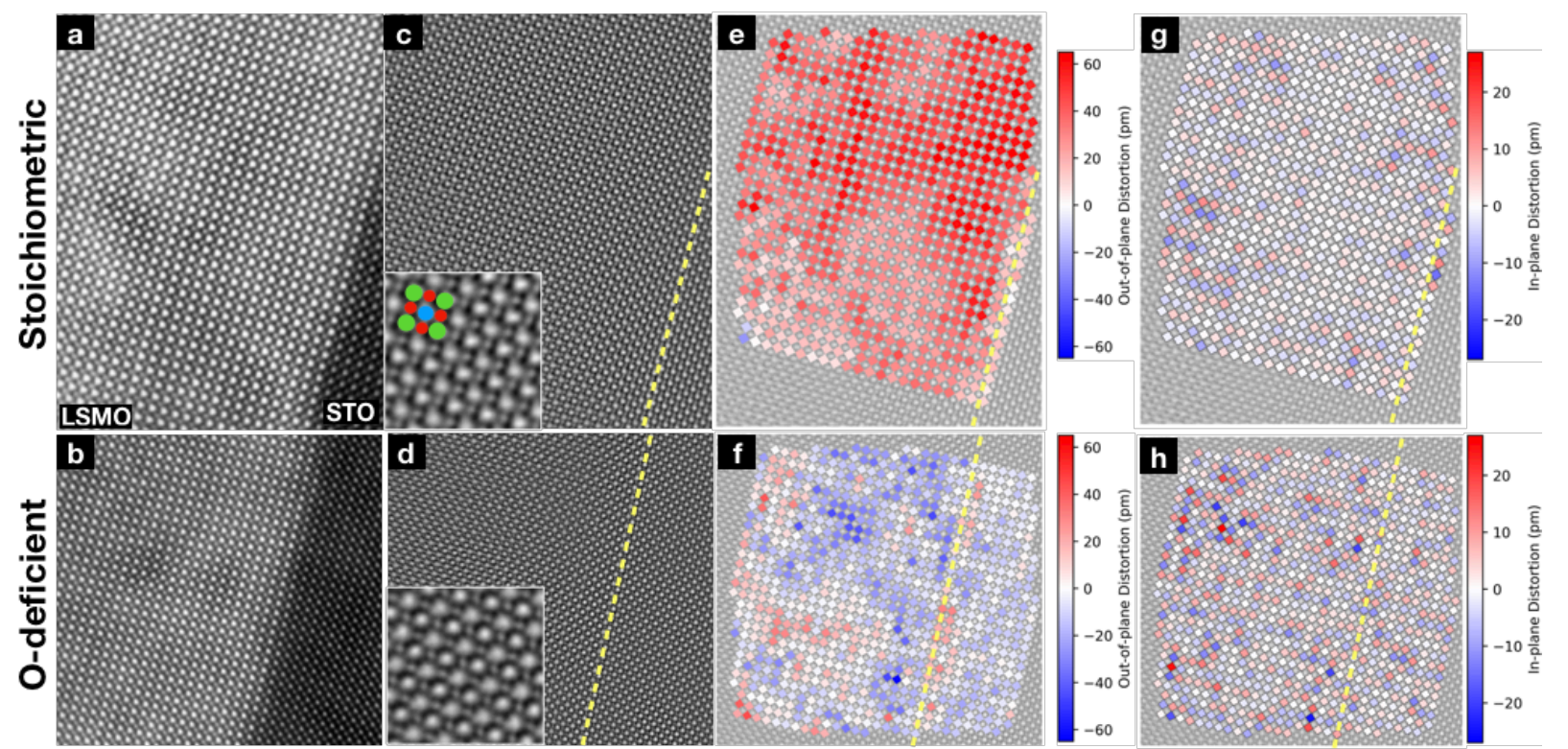

Figure 2. $(a, b)$ ADF images of the interface between stoichiometric (a) and oxygen-deficient (b) LSMO and STO. (c,d) Simultaneously acquired iDPC images. Blue, green, and red circles represent A-, B-and O-site atom columns, respectively. The dashed yellow lines mark the interface, (e,f) Out-of-plane component of octahedral distortion, measured perpendicular to the interface and $(\mathrm{g}, \mathrm{h})$ the in-plane component of octahedral distortion. 